\title{
Weakly bound atomic trimers in ultracold traps
}

\author{
M. T. Yamashita, ${ }^{1}$ T. Frederico, ${ }^{2}$ Lauro Tomio, ${ }^{3}$ and A. Delfino ${ }^{4}$ \\ ${ }^{1}$ Laboratório do Acelerador Linear, Instituto de Física da USP, 05315-970 São Paulo, Brazil \\ ${ }^{2}$ Departamento de Física, Instituto Tecnológico de Aeronáutica, Centro Técnico Aeroespacial, 12228-900 São José dos Campos, Brazil \\ ${ }^{3}$ Instituto de Física Teórica, Universidade Estadual Paulista, 01405-900 São Paulo, Brazil \\ ${ }^{4}$ Instituto de Física, Universidade Federal Fluminense, 24210-900 Niterói, Rio de Janeiro, Brazil
}

(Received 13 March 2003; published 15 September 2003)

\begin{abstract}
The experimental three-atom recombination coefficients of the atomic states ${ }^{23} \mathrm{Na}\left|F=1, m_{F}=-1\right\rangle$, ${ }^{87} \mathrm{Rb}\left|F=1, m_{F}=-1\right\rangle$, and ${ }^{85} \mathrm{Rb}\left|F=2, m_{F}=-2\right\rangle$, together with the corresponding two-body scattering lengths, allow predictions of the trimer bound-state energies for such systems in a trap. The recombination parameter is given as a function of the weakly bound trimer energies, which are in the interval $1<m(a / \hbar)^{2} E_{3}<6.9$ for large positive scattering lengths $a$. The contribution of a deep-bound state to our prediction, in the case of ${ }^{85} \mathrm{Rb}\left|F=2, m_{F}=-2\right\rangle$, for a particular trap, is shown to be relatively small.
\end{abstract}

DOI: 10.1103/PhysRevA.68.033406

PACS number(s): 32.80.Pj, 36.40.-c, 34.10.+x

The formation of molecules in ultracold atomic traps offers new and exciting possibilities to study the dynamics of condensates [1]. The formation of rubidium molecules ${ }^{87} \mathrm{Rb}_{2}$ in a bosonic condensate was reported, which allowed to measure its binding energy with unprecedented accuracy [2]. Ultracold sodium molecules ${ }^{23} \mathrm{Na}_{2}$ have also been formed through photoassociation [3]. However, nothing has been reported till now about the formation of molecular trimers in cold traps. The first information, one is led to ask, is about the magnitude of the binding energy of trimers in a cold trap. Two-body scattering lengths of trapped atoms are well known in several cases, as well as their closely related dimer binding energy. In the limit of large scattering lengths, it is necessary to know in addition one low-energy three-body observable to predict any other one. In this case, the detailed form of the two-body interaction is not important [4,5]. The recombination rate of three atoms in the ultracold limit, measured by atomic losses in trapped condensed systems, can supply the necessary information to estimate the trimer binding energy. For short-range interactions, the magnitude of the recombination rate of three atoms is mainly determined by the two-body scattering length $a$ [6]. However, it is important to remark that a dependence on one typical low-energy threebody scale $[4,5]$ still remains. Indeed, it is gratifying to note that all the works on three-body recombination, consistently, present a dependence on a three-body parameter in addition to the scattering length [7-9].

The aim of the present work is to report on how one can obtain the trimer binding energy of a trapped atomic system, from the three-body recombination rate and the corresponding two-body scattering length. For this purpose, we use a scale independent approach valid in the limit of large positive scattering lengths (or when the interaction range goes to zero), obtained from a renormalized zero-range three-body theory [4], which relates the recombination rate, the scattering length, and the trimer binding energy. Considering the experimental values of the recombination rates and scattering lengths given in Refs. [10-13], the method is applied to predict the trimer binding energies of ${ }^{23} \mathrm{Na} \mid F=1, m_{F}=$ $-1\rangle, \quad{ }^{87} \mathrm{Rb}\left|F=1, m_{F}=-1\right\rangle, \quad$ and $\quad{ }^{85} \mathrm{Rb}\left|F=2, m_{F}=-2\right\rangle$, where $\left|F, m_{F}\right\rangle$ is the respective hyperfine states of the total spin $F$. We note that the bound states considered here are in fact high-lying resonances, not true bound states, as they can decay into lower-lying channels.

The validity of our approach is restricted to sufficiently diluted gases, because all the scaling relations are derived for three isolated particles. Also, when the scattering length is tuned via external field in a trap, the parameters are different from the vacuum values, and consequently, our predictions only apply to that particular experimental conditions. For the trapped gases that we are analyzing, the diluteness parameter $\rho a^{3}$ (where $\rho$ is the gas density) should not be much larger than one, otherwise one needs to consider higher-order correlations between the particles. Indeed, we observe that, in general, for the analyzed condensed systems, the diluteness parameter is much smaller than one. Even in the case of ${ }^{85} \mathrm{Rb}$, where the considered scattering length is obtained via Feshbach resonance techniques [13], the diluteness parameter is about $1 / 2$.

Another relevant remark, pointed out in Ref. [14], is that the recombination into deep-bound states can affect the theoretical results that are based on calculation of this rate into shallow states alone. This additive contribution depends on one more constant, beyond the three-body scale. However, the fitted contribution of the recombination into deep-bound states is fortunately much smaller than the contribution of the shallow bound state, as found in the case of ${ }^{85} \mathrm{Rb}$ [14]. Such an evidence supports our estimation of trimer energies, when $a>0$, that are obtained by only considering the contribution of recombination rates into the shallow state.

The values of $a$ are usually defined as large with respect to the effective range $r_{0}$, such that $a / r_{0} \gg 1$. The low-energy three-boson system presents, in this limit, the Efimov effect [15], where an infinite number of weakly bound three-body states appears. The size of such states are much larger than the effective range. The limit $a / r_{0} \rightarrow \infty$ can be realized either by $a \rightarrow \infty$ with $r_{0}$ kept constant or by $r_{0} \rightarrow 0$ with $a$ constant. In the last case, the limit of a zero-range interaction corresponds to the Thomas bound-state collapse [16]. In this respect, the Efimov and Thomas limits are equivalent or different aspects of the same physics [17]. The Thomas-Efimov connection is also reviewed in Ref. [5]. In the limit $a / r_{0}$ 
$\rightarrow \infty$, the details of the interaction for the low-energy threebody system are contained in one typical three-body scale and the two-body scattering length (or the dimer bound-state energy $E_{2}$ ); they are enough to determine all three-body observables [4]. Considering, for example, the trimer binding energy $E_{3}$ as the three-body scale, any three-body observable $\mathrm{O}_{3}$ that has dimension of (energy) ${ }^{\beta}$, in the limit of $r_{0} \rightarrow 0$, can be expressed as

$$
O_{3}=E_{2}^{\beta} \mathcal{F}_{2}\left(E_{2} / E_{3}\right)=E_{3}^{\beta} \mathcal{F}_{3}\left(E_{2} / E_{3}\right)
$$

The dimensional factor in the above equation is chosen for convenience as $E_{2}$ or $E_{3}$. The scaling function in each case is $\mathcal{F}_{2}$ or $\mathcal{F}_{3}$. The existence of the scaling limit for zero-range interactions was verified in Refs. [18,19]. In practice, such a limit is approached by the excited state of the atomic trimer obtained in realistic calculations, allowing as well the theoretical interpretation of those excited states as Efimov states [19]. Here, we observe that the binding energy $E_{3}$ refers to the magnitude of the total energy of the bound system; the binding energy with respect to the two-body threshold is defined as $S_{3} \equiv E_{3}-E_{2}$.

The rate of three free bosons to recombine, forming a dimer and one remaining particle, is given in the limit of zero energy, by the recombination coefficient $[5,6]$

$$
K_{3}=\frac{\hbar}{m} a^{4} \alpha
$$

where $\alpha$ is a dimensionless parameter and $m$ is the mass of the atom. When $a>0$, the recombination parameter $\alpha$ oscillates between zero and a maximum value, which is a function of $a$, as shown in Refs. [7] ( $\alpha \leqslant 68.4)$, [8] ( $\alpha \leqslant 65)$, and [9] $(\alpha \leqslant 67.9)$. With amplitude $\alpha_{\max }$ and phase $\delta$, we can write it as [5]

$$
\alpha=\alpha_{\max } \sin ^{2}[1.01 \ln (a)+\delta],
$$

where $\delta$ depends on the interaction at short distances. The physics at short distances, in the three-boson system, is parametrized by one typical three-body scale, which we have chosen as the unknown trimer binding energy. So, by using the general scaling given by Eq. (1), one can explicitly express the functional dependence of $\alpha$ as $\alpha \equiv \alpha\left(\sqrt{E_{2} / E_{3}}\right)$, considering that for large scattering lengths we have $1 / a$ $=\sqrt{m E_{2} / \hbar^{2}}$. To exemplify the scaling form of $\alpha$, we can rewrite Eq. (3) such that the $\sqrt{E_{2} / E_{3}}$ dependence is explicit. Therefore,

$$
\alpha=\alpha_{\max } \sin ^{2}\left[-1.01 \ln \sqrt{\frac{E_{2}}{E_{3}}}+\Delta\left(\sqrt{\frac{E_{2}}{E_{3}}}\right)\right],
$$

where $\Delta\left(\sqrt{E_{2} / E_{3}}\right)=\delta-1.01 \ln \left(\sqrt{m E_{3} / \hbar^{2}}\right)$. Our next task is the calculation of the scaling function by using the renormalized subtracted Faddeev equations [4].

The three-boson recombination coefficient at zero energy is derived from the Fermi's golden rule as

$$
K_{3}=\frac{2 \pi}{\hbar}(2 \pi \hbar)^{9} \int \frac{d^{3} p}{(2 \pi \hbar)^{3}}\left|\mathcal{T}_{\mathrm{i} \rightarrow \mathrm{f}}\right|^{2} \delta\left(\frac{3}{4 m} p^{2}-E_{2}\right) \text {, }
$$

where $\mathcal{T}_{\mathrm{i} \rightarrow \mathrm{f}}$ is the transition amplitude between the initial $(i)$ and final $(f)$ momentum states, which are normalized as plane waves: $\langle\vec{r} \mid \vec{p}\rangle=\exp [-i(\vec{p} / \hbar) \cdot \vec{r}] /(2 \pi \hbar)^{3 / 2}$. The number of atoms $N$ in the condensed state decreases, due to the recombination process, as

$$
\frac{1}{N} \frac{d N}{d t}=-\frac{3}{3 !} K_{3} \rho^{2}
$$

For each recombination process three atoms are lost, justifying the factor 3 in the numerator. The factor 3 ! in the denominator appears only in case of condensed systems; it counts for the number of triples in such a state [11].

Considering the symmetrized scattering wave function for the initial state of three free particles, $\left|\Phi_{0}\right\rangle$ $=(1 / \sqrt{3}) \sum_{i=1}^{3}\left|\vec{q}_{i}, \vec{p}_{i}\right\rangle$, we obtain the transition amplitude, in terms of the Faddeev components of the three-body $T$ matrix $T_{i}(E)$, as

$$
\mathcal{T}_{\mathrm{i} \rightarrow \mathrm{f}}=\left\langle\vec{k}_{i} \Phi_{b}^{(j k)}\left|\left[T_{j}(E)+T_{k}(E)\right]\right| \Phi_{0}\right\rangle,
$$

where $(i, j, k)=(1,2,3)$ are cyclic permutations, and $E$ $\left[=3 q_{i}^{2} /(4 m)+p_{i}^{2} / m=3 k_{i}^{2} /(4 m)-E_{2}\right]$ is the energy of the scattering state. $\vec{q}_{i}$ is the Jacobi relative momentum of the particle $i$ with respect to the center of mass of particles $j$ and $k, \vec{p}_{i}$ is the relative momentum of $j$ with respect to $k$, and $\vec{k}_{i}$ is the relative momentum of the free particle $i$ in the final state. $\left|\Phi_{b}^{(j k)}\right\rangle$ is the normalized two-body bound-state wave function of the pair $j k$. The calculation of the Faddeev components is performed with the use of the subtracted approach given in Ref. [4], such that

$$
\begin{aligned}
T_{i}(E)= & t_{i}\left(E-\frac{3 q_{i}^{2}}{4 m}\right)\left\{1+\left[G_{0}^{+}(E)-G_{0}\left(E_{\mu}\right)\right]\right. \\
& \left.\times\left[T_{j}(E)+T_{k}(E)\right]\right\},
\end{aligned}
$$

where $E_{\mu} \equiv-\mu^{2} / m$ is the subtraction energy scale with $\mu$ a constant in momentum units. It is possible to vary $\mu$ without changing the physics of the theory as long as the inhomogeneous term of Eq. (8) is modified according to the renormalization group equations [20]. $t_{i}$ is the two-body $t$ matrix for the subsystem of particles $(j k)$. For $E=0$ and zero-range potential the corresponding matrix elements are given by [4]

$$
\left\langle\vec{p}^{\prime}\left|t\left(\frac{-3 q^{2}}{4 m}\right)\right| \vec{p}\right\rangle=\tau\left(\frac{-3 q^{2}}{4 m}\right)=\frac{1}{m \sqrt{3} \pi^{2}} \frac{1}{k-q+i \epsilon},
$$

where $k \equiv \sqrt{4 m E_{2} / 3}$. From Eqs. (8) and (9), and for $E=0$, the matrix elements of $T_{i}$ are given by

$$
\left\langle\vec{q}_{i} \vec{p}_{i}\left|T_{i}(0)\right| \overrightarrow{0} \overrightarrow{0}\right\rangle=\tau(0) \delta\left(\vec{q}_{i}\right)+2 \tau\left[-3 q_{i}^{2} /(4 m)\right] h\left(q_{i}\right),
$$




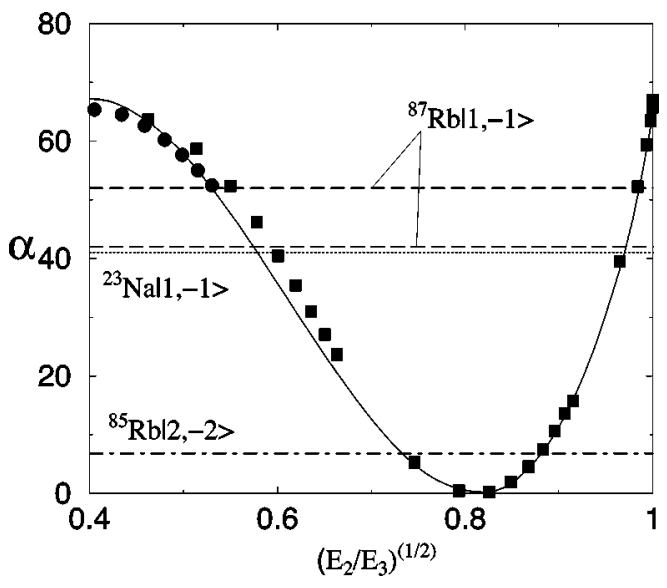

FIG. 1. The dimensionless recombination parameter $\alpha$ as a function of the ratio between the binding energies of the diatomic and triatomic molecules. Theoretical results: full circles (one triatomic bound state), solid line (two triatomic bound states), and full squares (three triatomic states). The lines indicate the center of experimental data, given in Table I, for ${ }^{23} \mathrm{Na},{ }^{87} \mathrm{Rb}$, and ${ }^{85} \mathrm{Rb}$. In case of ${ }^{85} \mathrm{Rb}$ we subtracted the contribution of the deep-bound state, which was reported in Ref. [14].

where the $s$ wave function $h(q)$ is the solution of

$$
\begin{aligned}
h(q)= & -\frac{\mu^{2}}{\sqrt{3} \pi^{2} k q^{2}\left(\mu^{2}+q^{2}\right)}-\frac{4}{\sqrt{3} \pi} \int_{0}^{\infty} \frac{d q^{\prime}}{q} \frac{q^{\prime} h\left(q^{\prime}\right)}{k-q^{\prime}+i \epsilon} \\
& \times \ln \left(\frac{q^{2}+q^{\prime 2}+q q^{\prime}}{q^{2}+q^{\prime 2}-q q^{\prime}} \frac{\mu^{2}+q^{2}+q^{\prime 2}-q q^{\prime}}{\mu^{2}+q^{2}+q^{\prime 2}+q q^{\prime}}\right) .
\end{aligned}
$$

The normalized two-body bound-state wave function, in the zero-range model, to be introduced in Eq. (7), is given by

$$
\left\langle\vec{p} \mid \Phi_{b}\right\rangle=\frac{1}{\pi} \sqrt{\frac{\hbar}{a}} \frac{1}{(\hbar / a)^{2}+p^{2}} .
$$

By considering the above equations, we obtain the final form of the recombination parameter:

$$
\begin{aligned}
\alpha= & \frac{8(2 \pi)^{8} m^{2}}{3 \sqrt{3}}\left(\frac{\hbar}{a}\right)^{5}\left|\mathcal{T}_{i \rightarrow f}\right|^{2} \\
= & 6 \sqrt{3}(8 \pi)^{2} \mid 1+\frac{16 \pi \hbar^{2}}{3 a^{2}} \int_{0}^{\infty} d q \frac{q h(q)}{k-q+\mathrm{i} \epsilon} \\
& \times\left.\ln \left(\frac{k^{2}+q^{2}+q k}{k^{2}+q^{2}-q k}\right)\right|^{2} .
\end{aligned}
$$

The numerical results for the recombination parameter are obtained from the solution of Eq. (11), for different values of $\mu$. When $\mu \rightarrow \infty$, the results approach the scaling limit $[18,19]$. Therefore, the theoretical results for $\alpha$ are shown in Fig. 1 as a function of the ratio $\sqrt{E_{2} / E_{3}}$. The calculations were performed in dimensionless units, such that all the momentum variables were rescaled in units of $\mu$ (in other words, $\mu=1$ in our calculations). So, the two-atom binding energy is decreased with respect to this scale. In that sense, the Thomas-Efimov states appear for $E_{2} / E_{\mu}$ going towards zero, which is equivalent of having $E_{2}$ fixed and $\mu \rightarrow \infty$. The parameter $\alpha$, shown in Fig. 1, is obtained as a function of the most excited trimer state. We have performed numerical calculations with at most three Efimov states. The full circles show the results when only one bound state exists. When $E_{2} / E_{\mu}$ allows two Efimov states, the results are represented by the solid curve, which is plotted against the energy of the excited state. With full squares we represent the results when $E_{2} / E_{\mu}$ allows three Efimov states. The scaling limit is well approached in our calculations. The maximum $\alpha$ occurs at the threshold $\left(E_{3}=E_{2}\right)$ and when $\left(E_{3} / E_{2}\right)^{1 / 2}=0.38$ [19]. So, according to this figure one obtains that $1<m(a / \hbar)^{2} E_{3}$ $<6.9$, a range consistent with Refs. [18,19,21]. The scaling limit for $\alpha$ has been obtained in Refs. [7,9], but without reference to the weakly bound triatomic molecular state.

In Fig. 1, we represent with horizontal lines the center of experimental values of $\alpha$, as given in Table I, for the hyperfine states ${ }^{23} \mathrm{Na}|1,-1\rangle,{ }^{87} \mathrm{Rb}|1,-1\rangle$, and ${ }^{85} \mathrm{Rb}|2,-2\rangle$. Using the measured values of $\alpha_{\text {expt }}$ one obtains, from the universal scaling plot, two weakly bound triatomic molecular states, denoted by $E_{3}$ and $E_{3}^{\prime}$, consistent with these values.

TABLE I. For the atomic species ${ }^{A} Z\left|F, m_{F}\right\rangle$, given in the first column, we present in the sixth and seventh columns our predicted trimer binding energies, with respect to the threshold, $S_{3} \equiv\left(E_{3}-E_{2}\right)$ and $S_{3}^{\prime} \equiv\left(E_{3}^{\prime}\right.$ $-E_{2}$ ), considering the central values of the experimental dimensionless recombination parameters $\alpha_{\text {expt }}$ (given in the fourth column). It is also shown that the corresponding two-body scattering lengths $a$ (second column), the diluteness parameters $\rho a^{3}$ (third column), and the dimer binding energies $E_{2}$ (fifth column). For ${ }^{87} \mathrm{Rb}|1,-1\rangle$, the recombination process was obtained in Ref. [11] for noncondensed $(*)$ and condensed $\left({ }^{\dagger}\right)$ trapped atoms.

\begin{tabular}{lcccccc}
\hline \hline${ }^{A} Z\left|F, m_{F}\right\rangle$ & $a(\mathrm{~nm})$ & $\rho a^{3}$ & $\alpha_{\text {expt }}$ & $E_{2}(\mathrm{mK})$ & $S_{3}(\mathrm{mK})$ & $S_{3}^{\prime}(\mathrm{mK})$ \\
\hline${ }^{23} \mathrm{Na}|1,-1\rangle$ & 2.75 & $6 \times 10^{-5}$ & $42 \pm 12[10]$ & 2.85 & 4.9 & 0.21 \\
${ }^{87} \mathrm{Rb}|1,-1\rangle$ & 5.8 & $1 \times 10^{-5}$ & $52 \pm 22^{*}[11]$ & 0.17 & 0.39 & 0.005 \\
${ }^{87} \mathrm{Rb}|1,-1\rangle$ & 5.8 & $1 \times 10^{-4}$ & $41 \pm 17^{\dagger}[11]$ & 0.17 & 0.30 & 0.013 \\
${ }^{87} \mathrm{Rb}|2,2\rangle$ & 5.8 & $4 \times 10^{-5}$ & $130 \pm 36[12]$ & 0.17 & & \\
${ }^{85} \mathrm{Rb}|2,-2\rangle$ & 211.6 & 0.5 & $7.84 \pm 3.4[1,13]$ & $1.3 \times 10^{-4}$ & $1.14 \times 10^{-4}$ & $3.8 \times 10^{-5}$ \\
\hline \hline
\end{tabular}


Considering the center of experimental values of $\alpha$, our predicted values for $E_{3}$ and $E_{3}^{\prime}$ are given in Table I in millikelvin. We are also giving in the table the corresponding known values of the scattering lengths. The range for the predicted values can be easily estimated from Fig. 1, considering the corresponding errorbars in $\alpha_{\text {expt }}$. See also Ref. [22], for a recent experiment with ultracold thermal gas of ${ }^{133} \mathrm{Cs}|3,3\rangle$, where the obtained values of $\alpha_{\max }$, considering their systematic error limits, are in good agreement with theory.

One observe that the trap diluteness parameter is smaller than one in all the cases. For ${ }^{85} \mathrm{Rb}|2,-2\rangle$, we study a case corresponding to $K_{3} \approx 3.5 \pm 1.5 \times 10^{-23} \mathrm{~cm}^{6} / \mathrm{s}$, extracted from Fig. 2(c) of Ref. [13], measured in an ultracold noncondensed gas with external field $B=156 \mathrm{G}$. This value of $B$ corresponds to $a=4000 a_{0}$ ( $a_{0}$ is the Bohr radius) (see Claussen et al. [1]). As the resulting value of $\alpha$ is quite small for ${ }^{85} \mathrm{Rb}|2,-2\rangle$, one should expect a more significant contribution from the deep-bound state. Thus, we found instructive to subtract such a contribution from $\alpha_{\text {expt }}$, which is about $1 \mu$, as found in Ref. [14]. However, the resulting effect in the determination of the trimer energy is not so dramatic, as seen in Fig. 1. The experimental value of $\alpha$ for ${ }^{87} \mathrm{Rb}|2,2\rangle$ does not appear in the figure, as it is well above the maximum. By increasing the value of $a$ from $5.8 \mathrm{~nm}$ to $6.8 \mathrm{~nm}$ we can make the experimental value consistent with our scaling limit approach. We also point out that the trimer can only support $E_{3}$ or $E_{3}^{\prime}$, not both simultaneously [19].

In our predictions for the trimer's energies, except for ${ }^{85} \mathrm{Rb}$, we have disregarded the possible much smaller contribution of the recombination rate into deep-bound states for $a>0$, considering only recombinations into shallow states. When the recombination into deep-bound states is taken into account, the curve in Fig. 1 is moved upward by an unknown amount. But, using the value found in Ref. [14], this contribution is hardly going to affect the extracted values for the trimer's binding energies, given in Table I. It seems natural that if one were to measure the recombination rate as a func- tion of an applied magnetic field, leading to a Feshbach resonance, one perhaps could be able to fix this additional contribution and determine the trimer binding energy. This additional contribution may help to explain part of the measured value of $\alpha$ for ${ }^{87} \mathrm{Rb}|2,2\rangle$.

In summary, in the present work, we derived the scaling dependence of the recombination parameter as a function of the ratio between the energies of the atomic dimer and the most excited trimer states. The scaling function tends to a universal function in the limit of zero-range interaction or infinite scattering length. The maximum of the recombination rate comes at the threshold for the appearance of a bound triatomic molecule. In the cases of diluted gases of ${ }^{23} \mathrm{Na}|1,-1\rangle,{ }^{87} \mathrm{Rb}|1,-1\rangle$, and ${ }^{85} \mathrm{Rb}|2,-2\rangle$, we use the scaling function, with the corresponding known experimental values of the recombination rates and two-atom scattering lengths, to predict the binding energies of weakly bound trimers in ultracold traps. We stress that the possible contribution of a deep-bound state in our predictions is expected to be relatively small, as verified for ${ }^{85} \mathrm{Rb}|2,-2\rangle$, in a particular trap. We also note that for ${ }^{85} \mathrm{Rb}|2,-2\rangle$ the diluteness parameter is about 0.5 , a value that may be considered near the limit of validity of the present approach, which does not include higher-order correlations between the particles.

Finally, we would like to remark that, at a first sight, one could think that formation of trimers requires four-body collisions, which are very unlikely unless the density is high. However, the recent experimental results given in Ref. [1] indicate formation of molecules in the trap, as also discussed in Ref. [23]. Therefore, other collision processes such as dimer-dimer or dimer and two atoms could also lead to trimer formations, enhancing the possibility of producing trimers in a trapped ultracold gas.

We thank V. S. Filho and A. Gammal for discussions. This work was partially supported by Fundação de Amparo à Pesquisa do Estado de São Paulo and Conselho Nacional de Desenvolvimento Científico e Tecnológico.
[1] N.R. Claussen, E.A. Donley, S.T. Thompson, and C.E. Wieman, Phys. Rev. Lett. 89, 010401 (2002); E.A. Donley, N.R. Claussen, S.T. Thompson, and C.E. Wieman, Nature (London) 417, 529 (2002).

[2] R.H. Wynar, R.S. Freeland, D.J. Han, C. Ryu, and D.J. Heinzen, Science 287, 1016 (2000).

[3] C. McKenzie et al., Phys. Rev. Lett. 88, 120403 (2001).

[4] S.K. Adhikari, T. Frederico, and I.D. Goldman, Phys. Rev. Lett. 74, 487 (1995); S.K. Adhikari and T. Frederico, ibid. 74, 4572 (1995).

[5] E. Nielsen, D.V. Fedorov, A.S. Jensen, and E. Garrido, Phys. Rep. 347, 373 (2001).

[6] P.O. Fedichev, M.W. Reynolds, and G.V. Shlyapnikov, Phys. Rev. Lett. 77, 2921 (1996).

[7] E. Nielsen and J.H. Macek, Phys. Rev. Lett. 83, 1566 (1999).

[8] B.D. Esry, C.H. Greene, and J.P. Burke, Jr., Phys. Rev. Lett. 83, 1751 (1999).
[9] P.F. Bedaque, E. Braaten, and H.-W. Hammer, Phys. Rev. Lett. 85, 908 (2000).

[10] D.M. Stamper-Kurn, M.R. Andrews, A.P. Chikkatur, S. Inouye, H.-J. Miesner, J. Stenger, and W. Ketterle, Phys. Rev. Lett. 80, 2027 (1998).

[11] E.A. Burt, R.W. Ghrist, C.J. Myatt, M.J. Holland, E.A. Cornell, and C.E. Wieman, Phys. Rev. Lett. 79, 337 (1997).

[12] J. Söding et al., Appl. Phys. B: Lasers Opt. B69, 257 (1999).

[13] J.L. Roberts, N.R. Claussen, S.L. Cornish, and C.E. Wieman, Phys. Rev. Lett. 85, 728 (2000).

[14] E. Braaten and H.-W. Hammer, Phys. Rev. Lett. 87, 160407 (2001).

[15] V. Efimov, Phys. Lett. B 33, 563 (1970); Comments Nucl. Part. Phys. 19, 271 (1990).

[16] L.H. Thomas, Phys. Rev. 47, 903 (1935).

[17] S.K. Adhikari, A. Delfino, T. Frederico, I.D. Goldman, and L. Tomio, Phys. Rev. A 37, 3666 (1988); S.K. Adhikari, A. 
Delfino, T. Frederico, and L. Tomio, ibid. 47, 1093 (1993).

[18] A.E.A. Amorim, T. Frederico, and L. Tomio, Phys. Rev. C 56, R2378 (1997).

[19] T. Frederico, L. Tomio, A. Delfino, and A.E.A. Amorim, Phys. Rev. A 60, R9 (1999); A. Delfino, T. Frederico, and L. Tomio, Few-Body Syst. 28, 259 (2000); J. Chem. Phys. 113, 7874 (2000).

[20] T. Frederico, A. Delfino, and L. Tomio, Phys. Lett. B 481, 143
(2000).

[21] The given limit was also recently confirmed in E. Braaten, H.-W. Hammer, and M. Kusunoki, Phys. Rev. A 67, 022505 (2003).

[22] T. Weber, J. Herbig, M. Mark, H.-C. Naegerl, and R. Grimm, e-print Physics/0304052.

[23] T. Köhler, T. Gasenzer, and K. Burnett, Phys. Rev. A 67, 013601 (2003). 